\title{
A LabVIEW-Based Virtual Instrument System for Laser-Induced Fluorescence Spectroscopy
}

\author{
Qijun Wu, ${ }^{1,2}$ Lufei Wang, ${ }^{1}$ and Lily $\mathrm{Zu}^{1}$ \\ ${ }^{1}$ Department of Chemistry, Beijing Normal University, Beijing 100875, China \\ ${ }^{2}$ Department of Chemistry, Bijie University, Bijie 551700, China \\ Correspondence should be addressed to Qijun Wu, wuqijun1977@yahoo.com.cn
}

Received 30 July 2011; Accepted 28 September 2011

Academic Editor: Lu Yang

Copyright ( 2011 Qijun Wu et al. This is an open access article distributed under the Creative Commons Attribution License, which permits unrestricted use, distribution, and reproduction in any medium, provided the original work is properly cited.

\begin{abstract}
We report the design and operation of a Virtual Instrument (VI) system based on LabVIEW 2009 for laser-induced fluorescence experiments. This system achieves synchronous control of equipment and acquisition of real-time fluorescence data communicating with a single computer via GPIB, USB, RS232, and parallel ports. The reported VI system can also accomplish data display, saving, and analysis, and printing the results. The VI system performs sequences of operations automatically, and this system has been successfully applied to obtain the excitation and dispersion spectra of $\alpha$-methylnaphthalene. The reported VI system opens up new possibilities for researchers and increases the efficiency and precision of experiments. The design and operation of the VI system are described in detail in this paper, and the advantages that this system can provide are highlighted.
\end{abstract}

\section{Introduction}

Laser-induced fluorescence (LIF) is a technique that relies on the measurement of the excited state fluorescence that results from photoexcitation. The excitation source for molecular LIF is typically a tunable dye laser so that selective excitation and high resolution can be achieved. Combined with the supersonic-jet expansion technique, LIF has been used to study the excited state structures of molecules and the ultra-fast dynamics of molecular systems [1]. LIF has also been successfully applied for quantitative measurement of concentrations in combustion, plasma, spray, and flow phenomena, in some cases visualizing concentrations down to nanomolar levels [2].

Unlike other analytical spectroscopy techniques, where ready-to-use commercial spectrometers have everything for the sample detection in a package, laboratories usually design and set up the LIF experimental instruments by themselves in order to satisfy individual research needs. In addition to hardware installation, LIF also requires synchronized and automated control software for laser function, data acquisition, and communication between instruments $[3,4]$. Most laboratories develop homemade programs for their experiments using different programming languages, such as Visual C++, LabVIEW, Visual Basic, or Turbo Pascal, but text-based languages have the shortcomings of long developing cycles and difficulties in maintenance and expansion [5]. NI LabVIEW 2009 (National Instrument Inc., USA) is a graphical programming tool which can be employed to develop sophisticated measurements [6-8] and control $[9,10]$ systems using intuitive graphical icons and wires that resemble a flowchart instead of written lines of text [11-16].

In this paper, a virtual instrument system based on LabVIEW 2009 was developed for LIF experiments. This system can simultaneously control the scanning of a dye laser and a monochromator, the operation of a molecular pump, and data acquisition. Furthermore, it achieves real-time data processing and monitoring of individual instruments. An application of this program in the LIF spectroscopy examination of $\alpha$-methylnaphthalene is shown in this paper. Simple modifications for adapting this program to different LIF setups are also illustrated.

\section{Configuration of System Hardware}

There are two different kinds of spectra in LIF spectroscopy, dispersed spectra, and excitation spectra. The dispersed spectra are obtained with fixed excitation laser wavelength and the fluorescence spectrum is analyzed. In excitation spectra, the laser wavelength exciting the molecules to a 


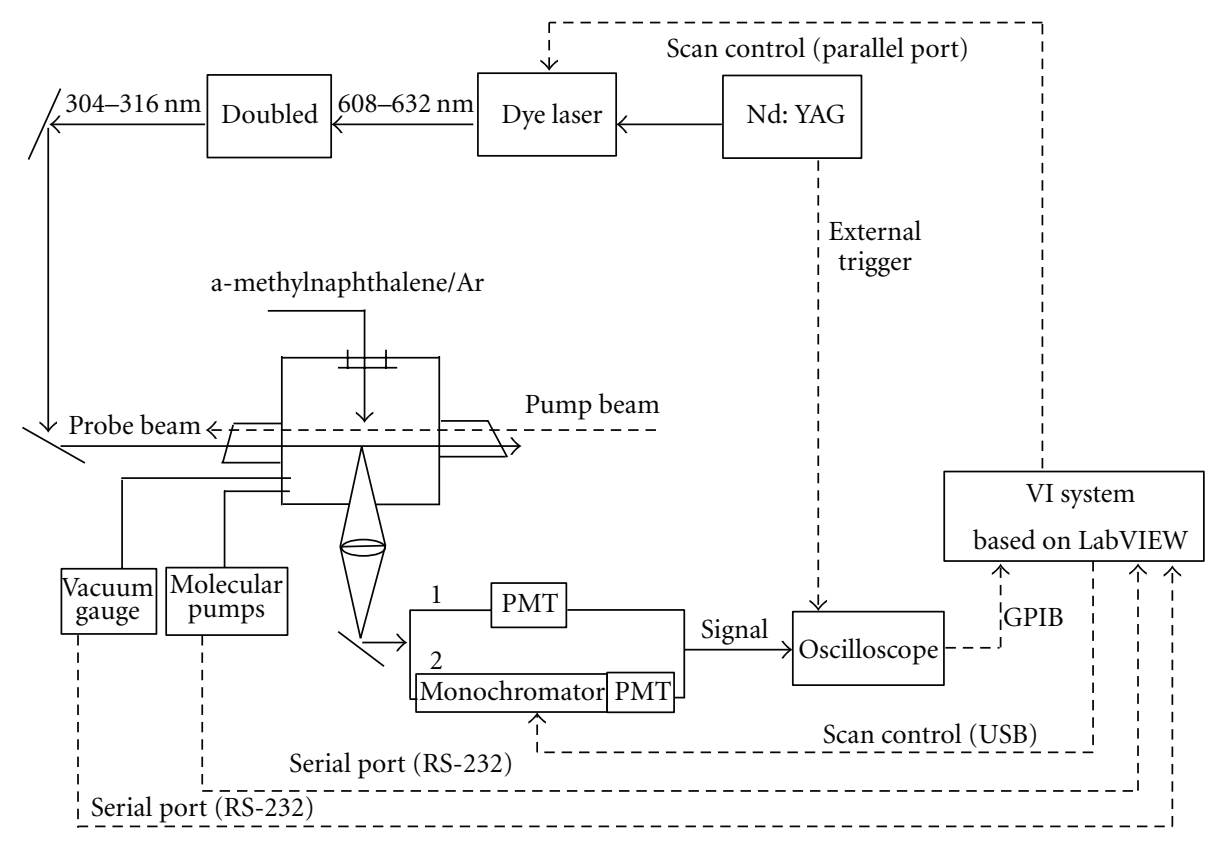

FIgURE 1: Hardware structure of VI System.

higher energy state is scanned and the total fluorescence is measured. Information on lower electronic states can be obtained by dispersed spectra, while the excitation spectra reveal parameters (energy levels, structure information, etc.) of upper level electronic states.

A conventional experimental setup for obtaining laserinduced fluorescence excitation spectra and dispersed spectra are depicted in Figure 1. The sample vapor is injected into a vacuum chamber, whose vacuum is maintained by a molecular pump and a mechanical pump, via a supersonic jet. The excitation laser beam (probe beam) is provided by a frequency doubled dye laser pumped by a Nd:YAG laser. The probe beam intercrosses the sample 10 to $15 \mathrm{~mm}$ downstream of the supersonic jet nozzle; when free radicals are studied, a pump laser beam propagates across the sample close to the nozzle and generates the free radicals. Fluorescence of the excited molecules is collected perpendicular to the probe beam by the optics and imaged onto the detection devices. For excitation spectra, total fluorescence is detected by PhotoMultiplier Tube (PMT) and converted into electronic signals, which are received and displayed in real time on an oscilloscope (synchronized by the triggers from the Nd:YAG laser). A General-Purpose Interface Bus (GPIB) card installed in the computer transfers data from the oscilloscope to the computer via the GPIB port, and data acquisition and processing are executed by the VI system. At the same time, the VI controls the dye laser scans by sending trigger pulses to the laser controller via the parallel port of the computer. The acquisition and control process for dispersed spectra are similar to those for excitation spectra. For dispersed spectra, the fluorescence signals are transmitted through a monochromator before being collected by the PMT. The VI system communicates with and controls the scan of the monochromator via the USB port in the monochromator. In addition, the VI system also communicates with the vacuum gauge via an RS232 port, and the system displays the in situ values of the vacuum. The VI system also builds up an interface to communicate with the operating program of the individual instruments (e.g., the dye laser and the molecular pump); specific operating programs are usually included with the instruments at the time of purchase.

\section{Programming}

Figure 2 shows the flow chart of the VI system that performs the LIF experiments and data processing tasks. It incorporates several subVIs, each one of which carries out a specific function, including data acquisition and display, data processing, as well as generating and saving the results.

There are three icons, Excitation Spectrum, Dispersed Spectrum, and Data Processing, on the front page of the virtual instrument system, each is dedicated to a specific action or experimental technique. When an icon is selected, the corresponding subVI program will be activated, and it will open the panel where specific tasks can be executed. For programming, an Event Structure for incident response is used to call the corresponding subVI [17]. When the subVI starts, the Event Structure waits until the precompiled program code is executed and the task is finished, then the Event Structure returns to wait for another event.

3.1. Program for Excitation Spectrum. To fulfill the requirements of LIF excitation spectra, the Excitation Spectrum subVI consists of fluorescence data acquisition, data display and saving, vacuum monitoring, communication with other application modules, and synchronization of laserwavelength-scan subVIs. 


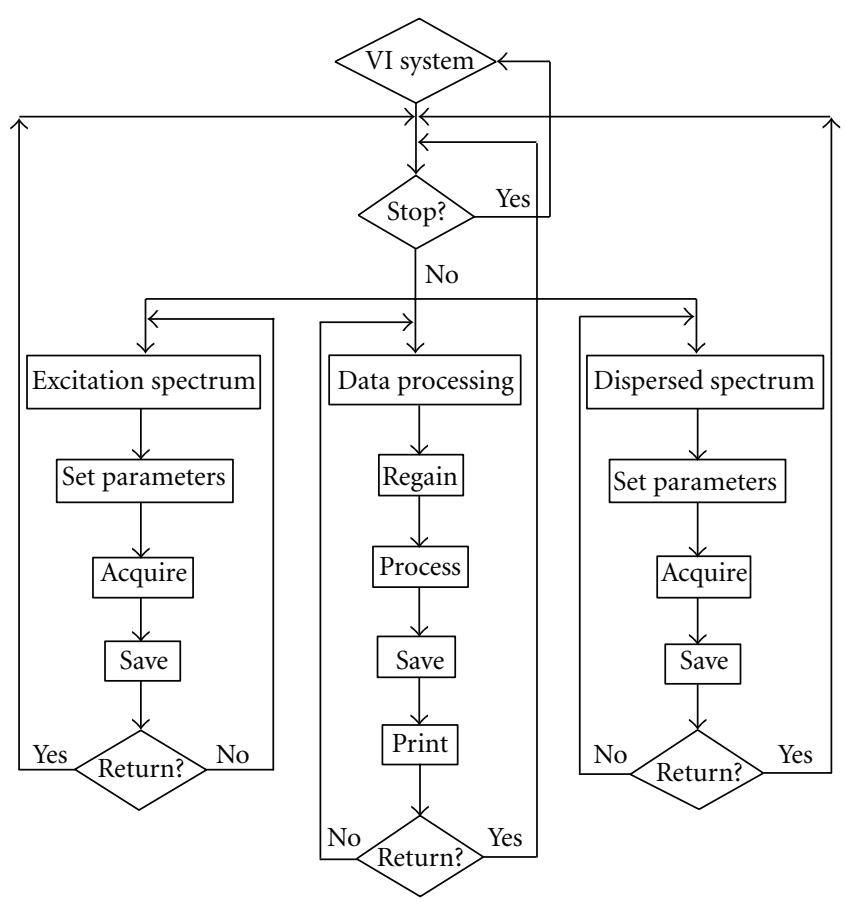

Figure 2: Flow chart of VI system.

3.1.1. Data Acquisition and Processing. In the experiment, the fluorescence signal is collected by the PMT and transferred to a TDS3032B oscilloscope. The TDS3032B oscilloscope supports the GPIB interface communication with a computer where the interface is an eight-bit parallel bus that provides high-speed data transfer and multiple-instrument control [18]. Detailed programming is shown in the upper left Flat Sequence Structure of Figure 3. We invoked the GPIB Write function from the LabVIEW Instrument $I / O$ function to set the GPIB address of the oscilloscope and specify the data parameters to be transferred from the oscilloscope, including the portion of the waveform that users want to transfer, the waveform source, data format, and the number of bytes per data point. We then invoked the GPIB Read function and wired the corresponding GPIB address of the oscilloscope to the GPIB Read, and also specified the number of bytes the function read from the oscilloscope. Finally, we called the function nodes Math Pattern, String Subset, Decimate 1D Array, and Join Numbers to receive waveform data from the oscilloscope and display it in situ in the front panel waveform graph. Data from different oscilloscope channels (CH1, CH2, or Dual CHs) can be acquired by wiring selected terminals (in a combo box) to the case structure prior to data acquisition. For the end-user, the above process is easily achieved by choosing the channel from the pull-down list on the front panel (Figure 4) and clicking on the Acquire button.

There are two sequential subdiagrams in the upper right case structure of Figure 3. The left one performs the analysis and processing of the fluorescence data obtained from the TDS3032B oscilloscope at a particular laser wavelength. Four cursors were created, and property nodes were set to acquire the cursor's horizontal axis positions on a waveform graph and to define the gate width. We then used the Array Subset to return two portions of fluorescence data between the two sets of cursors. On the front panel, users can directly drag the cursors to where they want, or they can simply set positions in the cursor legend to choose the gate on the graph. Integrals of data in both gates are computed separately using the $1 D$ Numeric Integration. $v i$, in which the integration method is the Trapezoidal Rule. The difference between the two gate integrals is calculated for the purpose of background subtraction. Finally, the fluorescence intensity and the current wavelength are sent to the XY Graph for realtime spectroscopy display. The front panel of the fluorescence Excitation Spectrum is shown in Figure 4, in which Choose Gates is shown in the upper portion and Spectroscopy Display in the lower part.

As soon as the data processing is complete, a TTL trigger is sent to step the dye laser to the next wavelength. TTL subVI, shown in the right sequential diagram in the case structure of Figure 3, calls the Outport.vi in Port I/O, which specifies a parallel port address for sending the trigger pulse and also defines the pulse width. A BNC cable is made with the inner core wired to the specified TTL pulse pin of the computer parallel port, and the outside shield wired to the ground pin. Thus, a TTL signal can be sent to the dye laser control unit from the computer parallel port when a data collection cycle is completed and the laser is stepped to the next scan wavelength. The settings for start position, end position, and step size of the laser wavelength scan must be identical in our VI program and in the laser control software. The TTL subVI is placed in a comparison case structure. If the current position is smaller than the end position, the comparison function will return a False value to initiate the program TTL.vi which will move the laser to the next wavelength to start a new data acquisition and processing cycle. If the current position is greater than or equal to the end position, the True case gives a message dialog box indicating that data acquisition (DAQ) is finished. Subsequently, data can be saved by employing the Write to Spreadsheet file and the Build Array and Transpose 2D Array nodes. This task is completed by clicking on the save button on the front panel and the fluorescence data will be saved according to the file name entered. The data acquisition process can be stopped at anytime by clicking the Acquire button again (in case the user wants to interrupt the experiment). An abort function is also programmed using the Stop node in the Application Control function, so users can terminate the whole VI system by clicking on the Abort-Execution button in the upperright corner (shown in Figure 4). For convenience, the help function of the program can be shown by clicking on the Help button on the front panel.

\subsubsection{Vacuum Monitoring and Accessing Other Application} Software. The program for the real-time display of the vacuum gauge is shown in the lower-left parallel loop of Figure 3. To communicate with the vacuum gauge, the Serial function templates in the LabVIEW Instrument I/O can be selected. Parameters such as VISA resource name, baud rate, data bits, and parity in the VISA Configure Serial Port are set according to the vacuum gauge communication protocol $[19,20]$. The VISA write function sends command characters 


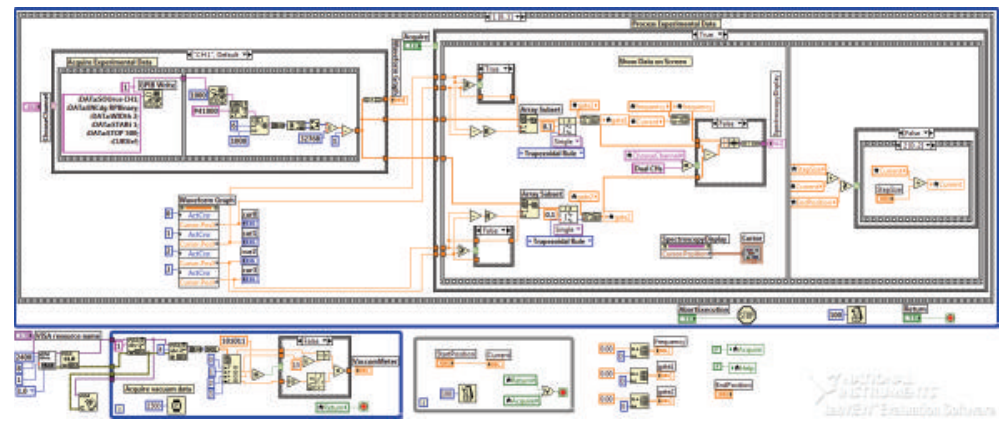

Figure 3: Program for excitation spectrum.

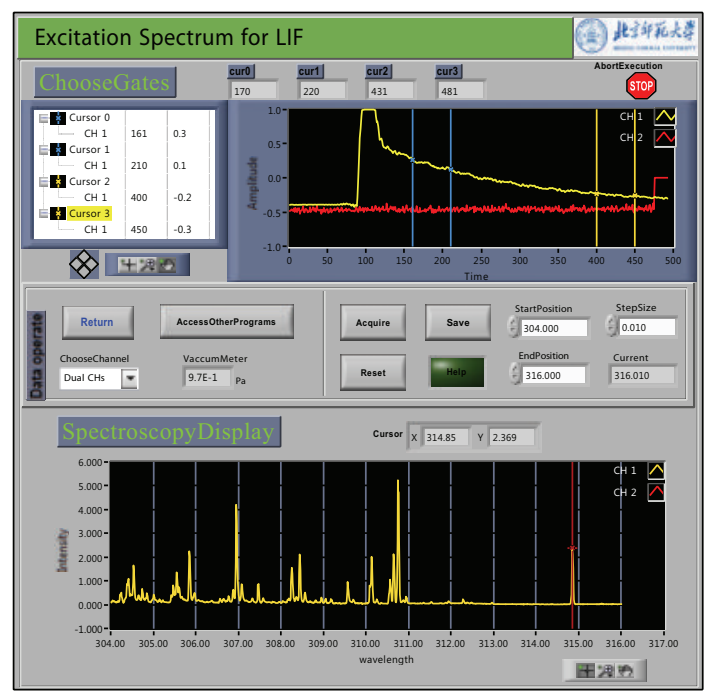

FIGURE 4: Front panel for excitation spectrum.

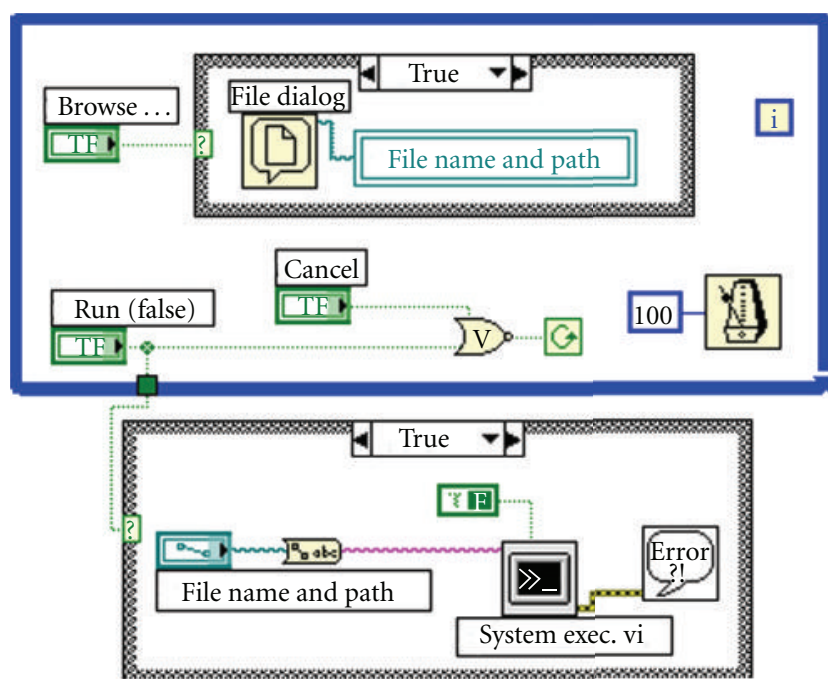

FIgure 5: SubVI for Access Other Programs. to the vacuum gauge and receives vacuum values in return. The nodes String to Byte Array, String to Single Precision Float, and Index Array transform the vacuum data into the required format and display the values on the front panel indicator.

For convenience, the VI system was designed to interface with the other applications that already exist for the individual instruments. By clicking on the Access-Other-Programs button on the excitation spectrum panel in Figure 4, users can select the application program by browsing the computer disks. Figure 5 shows the block diagram of the subVI for accessing other programs. The Browse Boolean command is wired to the upper case structure, which includes a File Dialog Express VI. The application can be selected when the file path is displayed on the front panel. When the user confirms the file selection, the application program is activated via the System Exec.vi shown in the lower case structure in Figure 5.

3.2. Program for Dispersed Spectra. A change in the dispersed spectrum program is the addition of synchronizing fluorescence DAQ with a monochromator wavelength scan instead of a dye laser scan. The 300i monochromator used in our dispersed LIF experiment was an Omni- $\lambda 3008$ spec- trometer (Beijing Zolix Instrument Company). Communication between the Omni- $\lambda 3008$ spectrometer and the computer is achieved via a USB port on the Omni- $\lambda 3008$ spectrometer. The Omni- $\lambda 3008$ spectrometer is operated by a ZolixOmniSpec ActiveX (OmniSpec; provided by the manufacturer). In the LabVIEW VI system, we invoke an ActiveX Container and created a ZolixOmniSpec ActiveX object; an Automation Open in the ActiveX functions palette is called, which returns an automation reference number and allows the specific functions programmed in the ZolixOmniSpec object to be accessed. Property nodes in the ActiveX palette are used for reading the USB port information. The Omni$\lambda 3008$ spectrometer USB serials string is found by calling the SearchZolixUSBDevice and GetZolixUSBSerial invoke methods, and then the string is wired to the USBSerials property node. The invoke commands (Open and Connect) are used for setting the connection status of the monochromator, and the status is displayed by the Boolean indicator on the front panel. The initial states of the Omni- $\lambda 3008$ monochromator components, such as the exit port, grating and current wavelength, are obtained by employing the GetExitPort, GetCurrentGrating, and CurrentWave methods, respectively. Next, different parameters are set according 


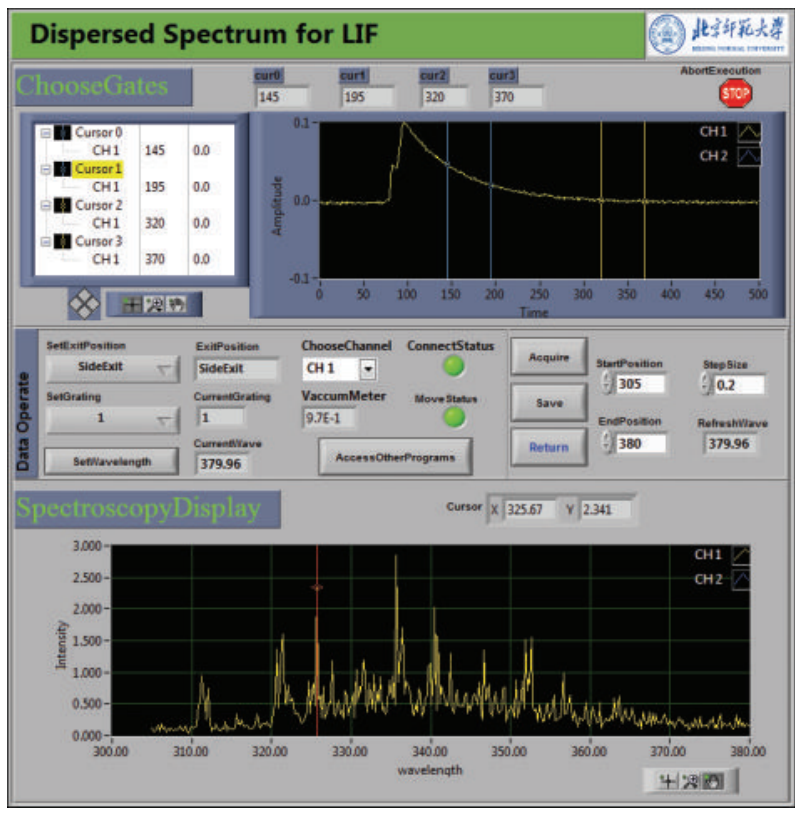

FIGURE 6: Front panel for dispersed spectrum.

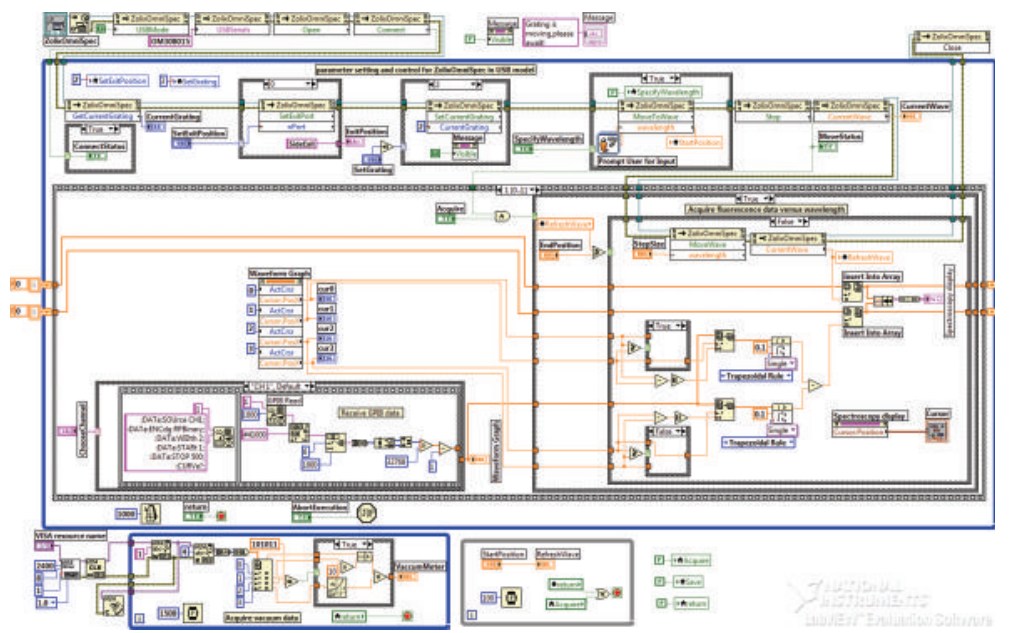

FIgURE 7: Program for dispersed spectrum.

to experimental requirements. This step involves the user's input of the experimental parameters by selecting from the pull-down menu on the front panel. The SetExitPort and SetCurrentGrating in the case structure return to the default state (which is set as Do Nothing) as soon as the commands are given to avoid sending looped control commands to the OmniSpec. The Prompt User for Input function in the Dialog and User Interface is called, and it prompts the user to input a specific wavelength, which is subsequently sent to the wavelength terminal in the MoveToWave method. After using the MoveToWave method, a Stop function is required to stop the grating from moving. The operation panel and program are shown in Figures 6 and 7.

A similar comparison case structure is applied here to step the monochromator scan wavelength as the one used in the Excitation LIF spectrum program. Identically to the excitation experiment, the start position, end position, step size of the monochromator scan, and the gate for fluorescence signal integration will await user input on front panel. Executed from the front panel, data acquisition will begin, and the real-time spectrum will be displayed in the $X Y$ Graph. Using the Save icon on the front panel, the experimental data can be stored in the computer via Write to Spreadsheet File.vi. An Automation Close is programmed in the VI system to disconnect the USB interface to the Omni$\lambda 3008$ and release the system resources when the Dispersed Spectrum subVI is closed.

3.3. Program for Data Processing. The data processing subVI (Figure 8) can conveniently redisplay and analyze previously saved data $[21,22]$. This subVI can automatically accomplish wavelength calibration, unit conversion, and peak and 


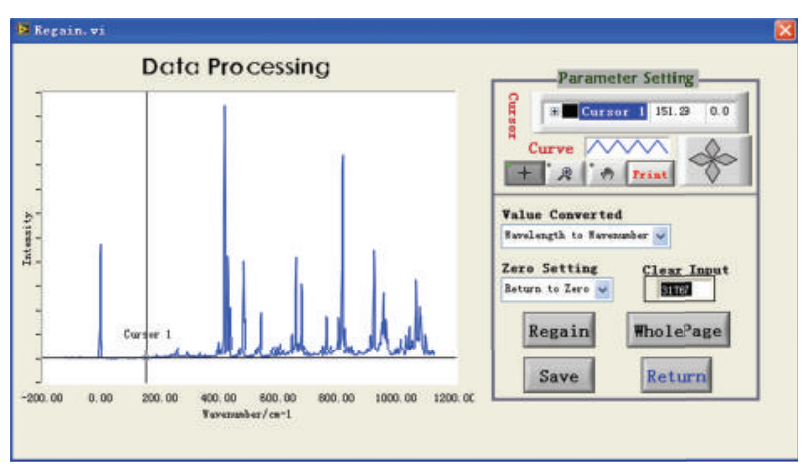

FIGURE 8: Front panel for data processing.

band-width reading of the spectrum curve. Users can also inspect each dataset using cursor move, data refresh, figure enlarge and diminish, and graph recover tools. In the VI programming, a File Dialog function will prompt the user to upload a data file. The Read From Spreadsheet File.vi and Index Array function will convert the wavelength and intensity data to $\mathrm{x}$ and $\mathrm{y}$ arrays, respectively. Wavelength unit conversion is realized by the case structures, and then the processed data will be sent to the $X Y$ Graph for display and saving by Write To Spreadsheet File.vi. Spectrum graph printing is achieved by using the relevant Report Generation functions.

\section{Application}

The VI system reported here has been used to take fluorescence spectra of molecules and free radicals in our laboratory. The excitation spectrum of a-methylnaphthalene is shown in Figure 4 (in wavelength, $\mathrm{nm}$ ) and Figure 8 (in wavenumber, $\mathrm{cm}^{-1}$ ). Dual $\mathrm{CHs}$ were used for data acquisition, one for studying the fluorescence signal and another for monitoring the laser power fluctuation using a photodiode. High resolution and signal-to-noise ratios were achieved in the spectra. The obtained excitation spectrum was consistent with previously reported results $[23,24]$ with spectral origin at $31767 \mathrm{~cm}^{-1}(314.80 \mathrm{~nm})$. The dispersed fluorescence spectrum of a-methylnaphthalene, shown in Figure 6, was obtained by exciting the most prominent band at the wavelength of $310.70 \mathrm{~nm}$. Dispersed spectra utilizing excitation of other bands $(306.50 \mathrm{~nm}$ and $314.80 \mathrm{~nm}$ ) were also obtained. Spectral analysis was conducted assisted by theoretical calculation and would be reported elsewhere [25].

\section{Conclusions}

A VI system for LIF spectroscopy based on LabVIEW 2009 has been developed. The VI system integrates a variety of laboratory equipment and controls the experiment data acquisition process using a single computer via GPIB, USB, RS232, and parallel ports. It achieves synchronous control of equipment and real-time fluorescence data acquisition. Data display, saving, and analysis can also be accomplished using the reported VI system. Furthermore, the VI system can monitor running of individual instruments and changing of vacuum and laser power in real time. Therefore, the users can timely judge, analyze, and process on-site information, and high resolution and signal-to-noise ratios of molecular or free radicals can be easily achieved in the spectra. Our approach has been to develop the VI system for general use in LIF experiments. The instruments used in this paper, such as the Nd:YAG laser (SureliteIII, Continuum), dye laser (NarrowScan, Radiant Dye), digital delay generator (DG535, Stanford), oscilloscope (TDS3032B, Tektronix), and PMT (CR110, Hamamatsu) are commonly used in many laboratories. When different instruments are to be used in an experiment, the users simply replace the communication method according to the communication protocol for the corresponding replacement instrument or change the file path in the Access-Other-Program subVI by following the instructions provided in the VI. The VI system has user-friendly front panel windows and is easy to operate. Help and Instructions implanted in the program will assist the user in becoming familiar with each application step.

The VI system was tested extensively and applied in the determination of the excitation spectrum and the dispersed spectrum of $\alpha$-methylnaphthalene with satisfactory experimental results. The VI system promotes and expands the capabilities of individual instruments, and it is an intelligent improving for existing hardware sources of laboratory. Lots of complicated functional operations are merely finished at the click of button on the VI system. The VI system not only avoids the manual error during the separate instrument control, data acquisition, and data processing, but ensures the accuracy and repeatability of experimental data and improves the efficiency of experiment. Furthermore, the VI system has a wide expandability of functions, minor modifications can be applied to a different LIF setups based on automatic measurement and control. The VI system will be provided upon request.

\section{Acknowledgments}

The authors are pleased to acknowledge financial support of this research by the National Natural Science Foundation of China (Grant no. 20673013). The authors also acknowledge grants from the Major State Basic Research Development Program (Grant no. 2007CB815206) and the Program for NCET.

\section{References}

[1] S. Gopalakrishnan, L. Zu, and T. A. Miller, "Rotationally resolved electronic spectra of the $\widetilde{B}-\tilde{X}$ transition in multiple conformers of 1-butoxy and 1-pentoxy radicals," Journal of Physical Chemistry A, vol. 107, no. 26, pp. 5189-5201, 2003.

[2] K. H. Katharina, "Quantitative laser-induced fluorescence: Some recent developments in combustion diagnostics," Applied Physics B Photophysics and Laser Chemistry, vol. 50, no. 6, pp. 455-461, 1990.

[3] L. Zhang, K. M. Callahan, D. Derbyshire, and T. S. Dibble, "Laser-induced fluorescence spectra of 4-methylcyclohexoxy radical and perdeuterated cyclohexoxy radical and direct 
kinetic studies of their reactions with O2," Journal of Physical Chemistry A, vol. 109, no. 41, pp. 9232-9240, 2005.

[4] J. Yang, X. Zhang, D. J. Clouthier, and R. Tarroni, "Heavy atom nitroxyl radicals. III. Identification of the $\mathrm{Cl}_{2} \mathrm{P}=\mathrm{S}$ free radical in the gas phase by laser spectroscopy and ab initio calculations," Journal of Chemical Physics, vol. 131, no. 20, pp. 204307-204313, 2009.

[5] H. Meng, J. Li, and Y. Tang, "The design and application of virtual ion meter based on LABVIEW 8.0," Review of Scientific Instruments, vol. 80, no. 8, Article ID 084101, 5 pages, 2009.

[6] S. Nuccio and C. Spataro, "Assessment of virtual instruments measurement uncertainty," Computer Standards and Interfaces, vol. 23, no. 1, pp. 39-46, 2001.

[7] G. C. Y. Chan and G. M. Hieftje, "A LabVIEW program for determining confidence intervals of Abel-inverted radial emission profiles," Spectrochimica Acta Part B, vol. 60, no. 11, pp. 1486-1501, 2005.

[8] F. J. Jiménez and J. De Frutos, "Virtual instrument for measurement, processing data, and visualization of vibration patterns of piezoelectric devices," Computer Standards and Interfaces, vol. 27, no. 6, pp. 653-663, 2005.

[9] D. Salehi and M. Brandt, "Melt pool temperature control using LabVIEW in Nd:YAG laser blown powder cladding process," International Journal of Advanced Manufacturing Technology, vol. 29, no. 3-4, pp. 273-278, 2006.

[10] G. Cautero, G. Paolucci, B. Brena et al., "A LabVIEW-based control system for a surface science experimental station," Measurement Science and Technology, vol. 5, no. 8, pp. 10021011, 1994.

[11] J. H. Moore, "Artificial intelligence programming with LabVIEW: Genetic algorithms for instrumentation control and optimization," Computer Methods and Programs in Biomedicine, vol. 47, no. 1, pp. 73-79, 1995.

[12] K. Yeung and J. Huang, "Development of a remote-access laboratory: A dc motor control experiment," Computers in Industry, vol. 52, no. 3, pp. 305-311, 2003.

[13] R. G. Castrejón and M. Duelk, "Using LabVIEW for advanced nonlinear optoelectronic device simulations in high-speed optical communications," Computer Physics Communications, vol. 174, no. 6, pp. 431-440, 2006.

[14] R. Y. Wang, J. A. Jarratt, P. J. Keay, J. J. Hawkes, and W. T. Coakley, "Development of an automated on-line analysis system using flow injection, ultrasound filtration and CCD detection," Talanta, vol. 52, no. 1, pp. 129-139, 2000.

[15] K. Brudzewski and J. Ulaczyk, "An effective method for analysis of dynamic electronic nose responses," Sensors and Actuators B, vol. 140, no. 1, pp. 43-50, 2009.

[16] G. Faraco and L. Gabriele, "Using LabVIEW for applying mathematical models in representing phenomena," Computers and Education, vol. 49, no. 3, pp. 856-872, 2007.

[17] “LabVIEW User Manual,” 2009, National Instruments Corporation.

[18] C. Wagner, S. Armenta, and B. Lendl, "Developing automated analytical methods for scientific environments using LabVIEW," Talanta, vol. 80, no. 3, pp. 1081-1087, 2010.

[19] W. B. Wang, J. Y. Li, and Q. J. Wu, "The design of a chemical virtual instrument based on LabVIEW for determining temperatures and pressures," Journal of Automated Methods and Management in Chemistry, vol. 2007, Article ID 68143, 7 pages, 2007.

[20] D. Nacapricha, N. Amornthammarong, K. Sereenonchai, P. Anujarawat, and P. Wilairat, "Low cost telemetry with PC sound card for chemical analysis applications," Talanta, vol. 71, no. 2, pp. 605-609, 2007.
[21] J. Ballesteros, J. I. F. Palop, M. A. Hernádez, R. Morales Crespo, and S. Borrego del Pino, "LabView virtual instrument for automatic plasma diagnostic," Review of Scientific Instruments, vol. 75, no. 1, pp. 90-93, 2004.

[22] F. Klocke, O. Dambon, U. Schneider, R. Zunke, and D. Waechter, "Computer-based monitoring of the polishing processes using LabView," Journal of Materials Processing Technology, vol. 209, no. 20, pp. 6039-6047, 2009.

[23] J. A. Warren, J. M. Hayes, and G. J. Small, "Symmetry reduction-vibronically induced mode mixing in the S1 state of $\beta$-methylnaphthalene," The Journal of Chemical Physics, vol. 80 , no. 5, pp. $1786-1790,1983$.

[24] N. A. Borisevich, G. G. D'yachenko, V. A. Petukhov, and M. A. Semenov, "Fine-structure spectroscopy of 2-methylnaphthalene cooled in a supersonic jet," Optics and Spectroscopy, vol. 101, no. 5, pp. 683-690, 2006.

[25] L. Wang, Q. Wu, and L. Zu, "Laser-induced fluorescence of 1-Methylnaphthalene in a supersonic jet expansion," Spectroscopy Spectral Analysis, vol. 31, no. 11, 4 pages, 2011. 


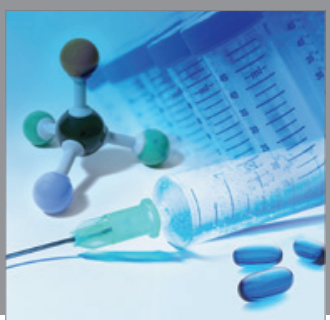

International Journal of

Medicinal Chemistry

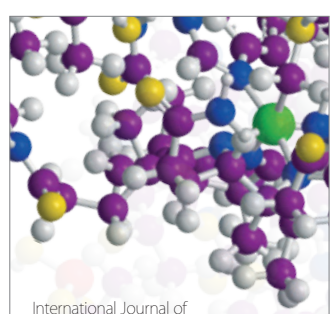

Carbohydrate Chemistry

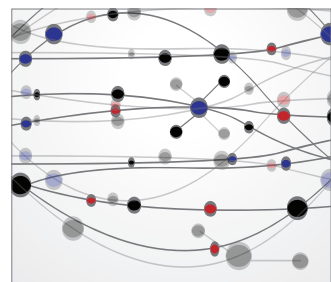

The Scientific World Journal
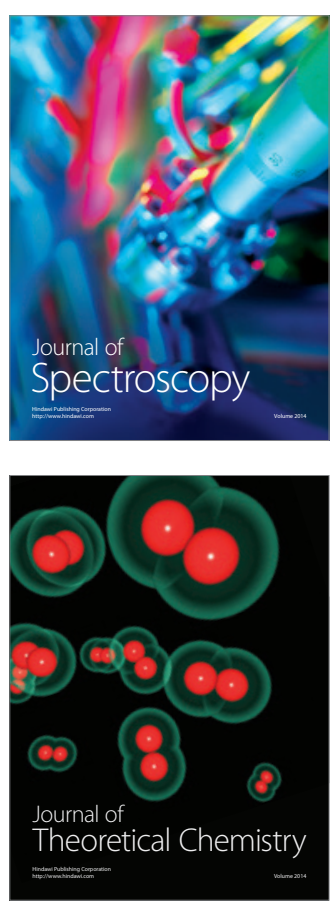
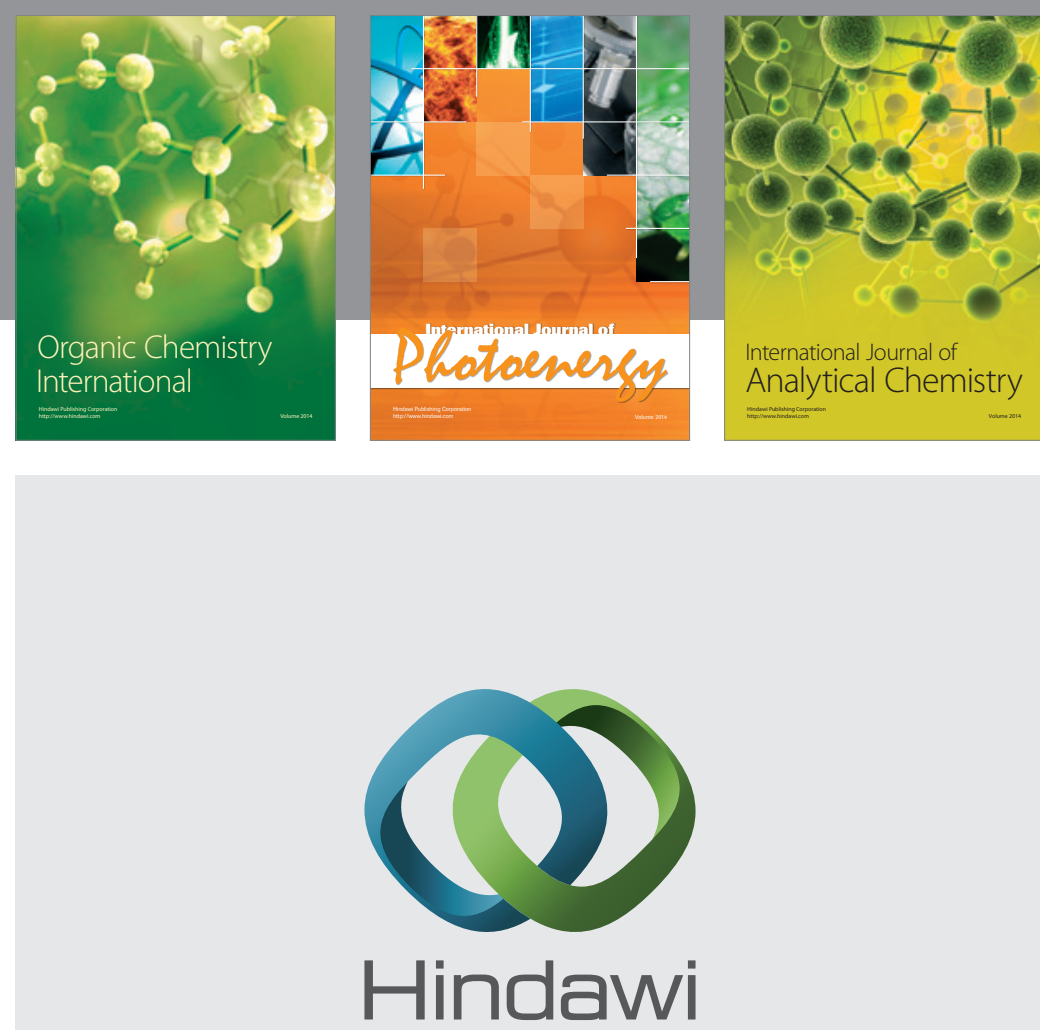

Submit your manuscripts at

http://www.hindawi.com
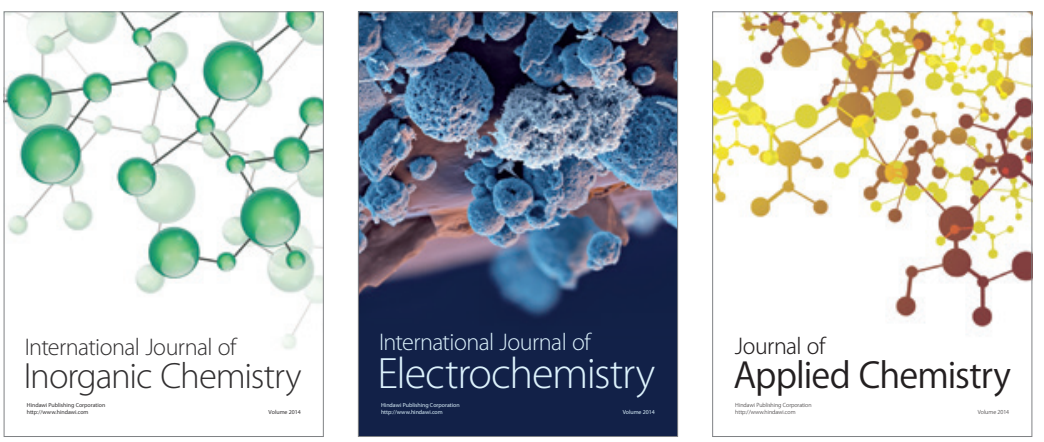

Journal of

Applied Chemistry
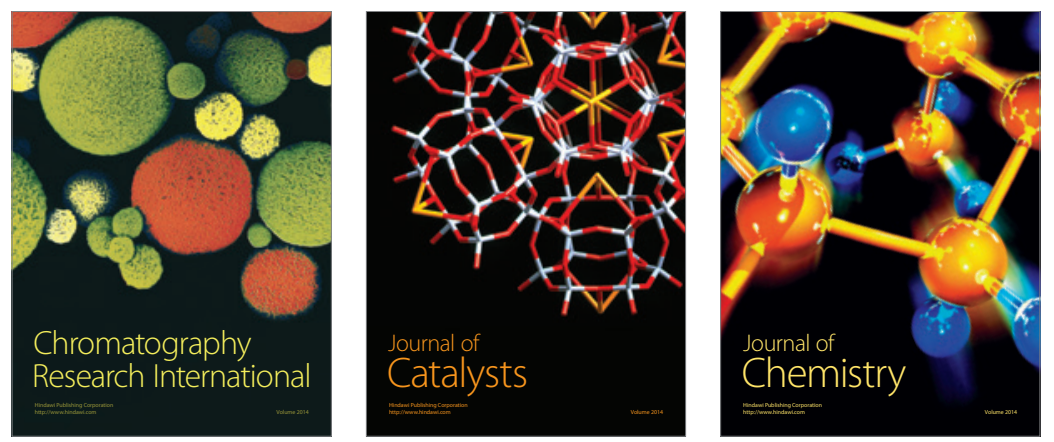
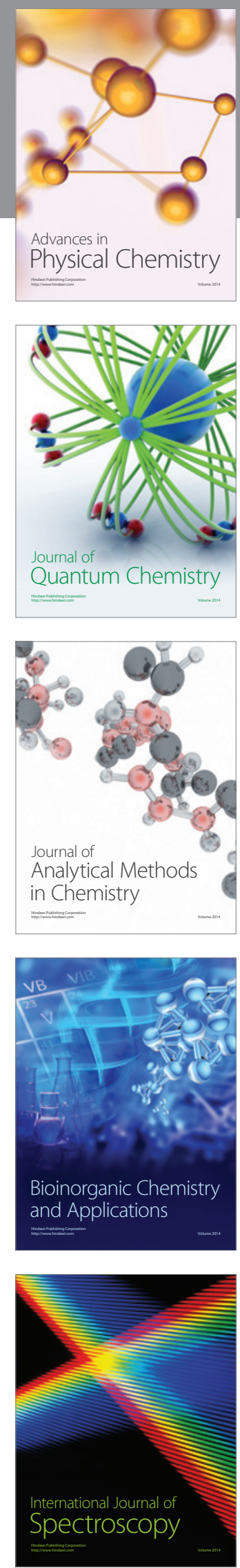